A N N A L E S Annales de Bretagne et des Pays de l'Ouest

\title{
HEUDRÉ, Bernard (dir.), DUFIEF, André (collaboration de), Souvenirs et observations de l'abbé François Duine
}

\section{Armelle Le Hüérou}

\section{(2) OpenEdition}

\section{Journals}

Édition électronique

URL : https://journals.openedition.org/abpo/1881

DOI : $10.4000 / a b p o .1881$

ISBN : 978-2-7535-1520-8

ISSN : 2108-6443

Éditeur

Presses universitaires de Rennes

Édition imprimée

Date de publication : 15 décembre 2010

Pagination : 214-216

ISBN : 978-2-7535-1309-9

ISSN : 0399-0826

Référence électronique

Armelle Le Hüérou, « HEUdRÉ, Bernard (dir.), Dufief, André (collaboration de), Souvenirs et observations de l'abbé François Duine », Annales de Bretagne et des Pays de l'Ouest [En ligne], 117-4 | 2010, mis en ligne le 17 janvier 2011, consulté le 22 juillet 2022. URL : http://journals.openedition.org/abpo/1881 ; DOI : https://doi.org/10.4000/abpo.1881 
BeAuduCEL, Christophe, L'imagerie populaire en Bretagne aux XVIII et $x^{e} X^{e}$ siècles, Rennes, PUR, 2009, 498 p.

Ce n'est pas d'hier que l'être humain a cherché à traduire sa pensée par le dessin. L'Église a su utiliser ce medium en couvrant les murs de ses sanctuaires de fresques édifiantes ou encore par les tableaux utilisés dans les retraites et les missions à partir du XVII ${ }^{\mathrm{e}}$ siècle. Ainsi, par l'intermédiaire de l'image et de la parole des ecclésiastiques, les masses analphabètes avaient accès à l'enseignement de la chaire. L'imagier n'était pas que peintre, il était aussi sculpteur et là encore le clergé sut utiliser ses talents en faisant exécuter en Basse-Bretagne de superbes calvaires pour la plupart aux $x v^{e}$ et $x v^{e}{ }^{e}$ siècles. Là encore, le but était le même, s'appuyer sur une représentation visuelle pour faire passer par l'oral un message pastoral.

À partir du XVIII siècle, ce sont encore des visées religieuses qui vont donner aux faiseurs et marchands d'images, sur papier cette fois, l'occasion de développer un commerce qui sera florissant. C'est ce sur quoi Christophe Beauducel vient d'effectuer une brillante recherche qu'il publie dans un ouvrage consacré à l'imagerie populaire en Bretagne aux XVIII et $\mathrm{XIX}^{\mathrm{e}}$ siècles. Dans un fort volume de près de 500 pages, l'auteur effectue tout d'abord un recensement des travaux de ses prédécesseurs sur ce sujet. II signale le véritable premier livre de référence paru en 1925 sous la plume de Pierre-Louis Duchartre (1894-1983) et René Saulnier (1869-1957) mais celui qu'il considère comme le plus remarquable, c'est celui de Maurice Jusselin sur l'imagerie chartraine, paru en 1957. Selon lui, la méthodologie de recherche, très innovante, constitue un modèle du genre. Fondée essentiellement sur l'analyse d'archives, elle restitue le parcours biographique et professionnel des imagiers et cartiers chartrains à partir du milieu du xvie siècle. Christophe Beauducel suit cet exercice exemplaire de recherche archivistique. II fouille dans toutes les sources disponibles notamment les registres de capitation et les divers documents fiscaux mais aussi, les minutes notariales, les inventaires après décès, les dossiers d'imprimeurs, les registres d'état-civil... autrement dit, il effectue un véritable travail de fourmi qui le conduit à retracer par le menu sur l'ensemble de la Bretagne historique les biographies de nombreux personnages, pour beaucoup peu connus ou méconnus, les fabricants d'images, les marchands sédentaires et les colporteurs, un travail qui n'avait jamais été effectué auparavant de manière exhaustive et avec autant de minutie.

Ainsi, une enquête rigoureuse lui permet de faire des découvertes d'importance. Il met en évidence le lien qui a existé entre la carte et l'image, les cartiers s'avérant être souvent à l'origine de l'imagerie. Les premières images furent en effet produites par des cartiers. II démontre l'influence d'acteurs extérieurs à la Bretagne et le rôle joué en particulier par ces Normands de Coutances qui furent les véritables pionniers en la matière dans notre pays. II signale aussi comment l'imagerie bretonne était dépendante des modèles extra-régionaux, français et étrangers, et aussi bien influencée par d'autres estampes populaires que par les tableaux des plus grands maîtres.

La recherche des images elles-mêmes l'a conduit à augmenter considérablement le corpus de documents connus à ce jour, passant d'une centaine à plus de quatre cents. C'est ce qui lui permet de constater aussi que l'ensemble des collections dont on dispose est constitué essentiellement de sujets religieux. II explique enfin pourquoi l'activité des imagiers a périclité au $x^{\mathrm{e}}{ }^{\mathrm{e}}$ siècle, notamment lorsqu'ils se sont mis à imiter les productions d'Épinal, négligeant par ailleurs de suivre l'évolution des techniques de reproduction de l'image.

L'ouvrage de Christophe Beauducel constitue donc un apport majeur dans la recherche à propos de ces images populaires. Peut-on, tout de même, se permettre 
de faire une ou deux petites remarques ou suggestions à son sujet? On aurait aimé en savoir un peu plus sur la clientèle, le public qui achetait ces images. De même, on aurait pu insister sur les lieux où l'on pouvait se procurer les images en dehors des échoppes des marchands des villes ou dans le sac des colporteurs. Une comparaison avec d'autres productions populaires, comme par exemple les chansons sur feuilles volantes, constituerait une piste susceptible d'éclairer ces questions et d'augmenter encore le corpus d'images. C'est une source qui ne semble pas avoir été exploitée en dehors des grandes planches en couleur de saints personnages, comme saint Cornely ou saint Cado. L'existence de chansons sur feuilles volantes éditées au XVII siècle à Quimper, notamment par Jean Perrier, et ornées de jolis bois gravés, laisse penser qu'on devrait pouvoir en trouver d'autres avant et après cette époque. En outre, fréquemment des gravures de frises et vignettes de différentes sortes figurent sur les chansons sur feuilles volantes en langue bretonne des XVIII et XIXe siècle. II serait aussi intéressant d'en dresser un catalogue pour identifier leur origine et enrichir le corpus.

L'auteur remarque la fréquence des gravures sur le thème notamment du Juif errant, de Geneviève de Brabant, de Damon et Henriette, le fils prodigue. C'est aussi le cas pour les chansons sur feuilles volantes en langue bretonne dont Joseph Ollivier signale dans son catalogue de nombreuses rééditions. Ce sont respectivement, 11, 14,5 et 9 rééditions de ces mêmes sujets mais on sait la fragilité de ces feuillets et on peut penser que leur nombre fut certainement plus élevé. À ce sujet, il ne serait pas inutile d'établir des statistiques concernant les images comportant des textes en langue bretonne, ce qui en ferait aussi une certaine spécificité régionale.

Enfin, les différentes utilisations de la gravure, ce qu'elle devient une fois achetée, ses diverses fonctions, mériteraient aussi une enquête peut-être plus poussée. Ce ne sont bien sûr que des suggestions et cet ouvrage, redisons-le, d'une lecture fort agréable, constitue à lui seul une somme qui fera date dans l'histoire de l'imagerie bretonne et prolonge en l'enrichissant ce qui fut un autre événement, la superbe exposition (et le catalogue) sur le sujet présentée par le Musée départemental breton à Quimper en 1992.

Daniel GIRAUDON

CRBC/Université de Bretagne Occidentale, Brest

Heudré, Bernard (dir.), Dufief, André (collaboration de), Souvenirs et observations de l'abbé François Duine, PUR, 2009, 348 p (cahier photo. 24 p.).

Le nom de l'abbé François Duine (1870-1924) est aujourd'hui surtout familier aux spécialistes d'hagiographie bretonne, qui s'appuient encore sur nombre des travaux érudits de cet esprit critique et clerc atypique. En dépit de leurs dates anciennes de publication, beaucoup d'entre eux, qu'il s'agisse de monographies, d'articles publiés aux Annales de Bretagne, ou dans d'autres revues savantes, demeurent dans ce domaine un point de départ obligé et stimulant ${ }^{4}$. La publication des quatre cahiers ${ }^{5}$ qui constituent l'actuel volume de ses Souvenirs et observations ne manquera pas d'élargir son lectorat, en intéressant aussi bien les amateurs de Mémoires que les historiens de la période contemporaine et ceux de l'Église, en particulier en Bretagne.

4. Cf. en dernier lieu, J.-C. Poulin, L'hagiographie bretonne du Haut Moyen Âge. Répertoire raisonné, Ostfildern (Thorbecke) 2009, p. 467.

5. Actuellement conservés à la BnF (Nouvelles acquisitions françaises 12997-13000). 